\title{
CONDICIONES DE TRABAJO DE LOS EGRESADOS DE ENFERMERÍA EN CARTAGENA
}

Zuleima Cogollo Milanés

Universidad de Cartagena Colombia

Adalgiza Polo Marques Universidad de Cartagena Colombia

Ana Cristina Guzmán Universidad de Cartagena Colombia

Edna Gómez Bustamante Universidad de Cartagena Colombia 
Panorama Económico, Vol. 27 - No. 1 (Enero - Marzo de 2019), pp. 105-115

Zuleima Cogollo Milanés

Adalgiza Polo Marques

Ana Cristina Guzmán

Edna Gómez Bustamante

JEL: J30, J80, J81

\title{
Condiciones de trabajo de los egresados de Enfermería en Cartagena \\ Resumen
}

El trabajo se considera el medio por el cual las personas obtienen recursos para alcanzar las metas propuestas, su desempeño depende de las condiciones que la organización ofrezca para la realización de sus actividades. Para los profesionales de enfermería el ambiente laboral es complejo, en especial si laboran en áreas asistenciales, por considerar que las actividades que llevan a cabo presentan una serie de riesgos derivados de la interacción con los sujetos de cuidado. En ese orden, se buscó identificar las condiciones de trabajo de los egresados de un programa de Enfermería de una universidad pública de Colombia. Para ello se diseñó un estudio descriptivo, con una muestra de 110 enfermeras, a las que se les aplicó una encuesta del Observatorio Laboral para la Educación, que indaga, características sociodemográficas, características del cargo, relaciones laborales, contratación y condición de seguridad laboral. Los resultados obtenidos lograron identificar que el $20 \%$ de las enfermeras tenía entre uno y dos años de desempeño profesional y $44 \%$ estaban contratadas por cooperativas de trabajo asociado. El 45\%, la mayoría reciben salarios entre $\$ 1^{\prime} 500.000$ y $\$ 2{ }^{\prime} 000.000$, 33\% entre 1'000.000 y menos 1'500.000, pese a estos bajos salarios y estas formas de contratación ilegales, el 95\% de los egresados considera que el trabajo ha contribuido a su crecimiento personal y el $62 \%$ se encuentra satisfecho con el empleo desempeñado y consideran, además, muy útil las competencias adquiridas durante su formación profesional. Sin embargo, las condiciones laborales, salariales y el escaso reconocimiento social, no son congruentes con la dedicación y el profesionalismo del acto de cuidar. Las enfermeras necesitan ser reivindicadas por el gobierno Nacional como un colectivo mayoritariamente femenino con desventaja de menores salarios frente a otros grupos de profesionales de la salud mayoritariamente masculinos.

Palabras clave: Profesional de enfermería, Condiciones de trabajo, servicios de salud.

\section{Conditions de travail des diplômés Infirmiers d'une université publique en Cartagena Résumé}

El trabajo se considera el medio por el cual las personas obtienen recursos para alcanzar las metas propuestas, su desempeño depende de las condiciones que la organización ofrezca para la realización de sus actividades. Para los profesionales de enfermería el ambiente laboral es complejo, en especial si laboran en áreas asistenciales, por considerar que las actividades que llevan a cabo presentan una serie de riesgos derivados de la interacción con los sujetos de cuidado. En ese orden, se buscó identificar las condiciones de trabajo de los egresados de un programa de Enfermería de una universidad pública de Colombia. Para ello se diseñó un estudio descriptivo, con una muestra de 110 enfermeras, a las que se les aplicó una encuesta del Observatorio Laboral para la Educación, que indaga, características sociodemográficas, características del cargo, relaciones laborales, contratación y condición de seguridad laboral. Los resultados obtenidos lograron identificar que el $20 \%$ de las enfermeras tenía entre uno y dos años de desempeño profesional y $44 \%$ estaban contratadas por cooperativas de trabajo asociado. El 45\%, la mayoría reciben salarios entre $\$ 1^{\prime} 500.000$ y $\$ 2$ '000.000, 33\% entre 1 '000.000 y menos 1'500.000, pese a estos bajos salarios y estas formas de contratación ilegales, el $95 \%$ de los egresados considera que el trabajo ha contribuido a su crecimiento personal y el $62 \%$ se encuentra satisfecho con el empleo desempeñado y consideran, además, muy útil las competencias adquiridas durante su formación profesional. Sin embargo, las condiciones laborales, salariales y el escaso reconocimiento social, no son congruentes con la dedicación y el profesionalismo del acto de cuidar. Las enfermeras necesitan ser reivindicadas por el gobierno Nacional como un colectivo mayoritariamente femenino con desventaja de menores salarios frente a otros grupos de profesionales de la salud mayoritariamente masculinos.

Mots clés: Infirmière professionnelle, conditions de travail, services de santé.

\section{Working conditions of Nursing graduates of a public university in Cartagena}

\begin{abstract}
Work is considered the means by which people obtain resources to achieve the proposed goals, their performance depends on the conditions that the organization offers to carry out its activities. For nursing professionals, the work environment is complex, especially if they work in healthcare areas, considering that the activities they carry out present a series of risks derived from the interaction with the care subjects. In this order, we sought to identify the working conditions of graduates of a nursing program from a public university in Colombia. For this, a descriptive study was designed, with a sample of 110 nurses, to whom a survey by the Labor Observatory for Education was applied, which investigates sociodemographic characteristics, job characteristics, labor relations, hiring and job security condition. The results obtained were able to identify that $20 \%$ of the nurses had between one and two years of professional performance and $44 \%$ were hired by associate work cooperatives. $45 \%$, most receive salaries between $\$ 1,500,000$ and $\$$ $2,000,000,33 \%$ between 1,000,000 and less 1,500,000, despite these low wages and these illegal forms of hiring, $95 \%$ of graduates consider that the Work has contributed to their personal growth and $62 \%$ are satisfied with the job they have performed and also consider the skills acquired during their professional training to be very useful. However, the working conditions, wages and the low social recognition are not consistent with the dedication and professionalism of the act of caring. Nurses need to be vindicated by the National government as a majority female group with a disadvantage of lower wages compared to other groups of mostly male health professionals.
\end{abstract}

Keywords: Nursing professional, working conditions, health services. 


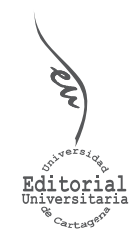

Artículo de Investigación

\section{Condiciones de trabajo de los egresados de Enfermería en Cartagena}

INFORMACIÓN DEL ARTÍCULO

Recepción del artículo: 15/09/2018

Concepto de evaluación: 23/10/2018

Aceptación del artículo: 30/11/2018
Zuleima Cogollo Milanés* Universidad de Cartagena, Colombia

Adalgiza Polo Marques Universidad de Cartagena, Colombia

Ana Cristina Guzmán Universidad de Cartagena, Colombia

Edna Gómez Bustamante Universidad de Cartagena, Colombia

\section{INTRODUCCIÓN}

A largo de la historia el trabajo ha sido considerado como una estrategia por medio de la cual las personas obtienen recursos para alcanzar las metas propuestas en su vida y su desempeño va depender muchas veces de las condiciones que la organización le preste para la realización de sus actividades, es por eso que las condiciones de trabajo son definidas como un conjunto variables intra y extralaborales que interactúan entre sí, que influyen en el desempeño y la salud del trabajador (Pico, 2001). Al mismo tiempo, el trabajo es una actividad de vastos alcances que se refleja e influye sobre casi todos los aspectos de la conducta humana, por lo que los individuos al desempeñar un trabajo no sólo trasladan a él sus habilidades intelectuales y motrices, sino también sus individualidades (Fernández y Paravic, 2003).

En el caso de los profesionales de la salud, el ambiente en que estos se desempeñan es un poco complejo, en especial si laboran en áreas asistenciales, teniendo en cuenta que las actividades que realizan; la cercanía con la muerte, el sufrimiento físico, psíquico, la incertidumbre de la recuperación o no, generan tensiones y riesgo para la salud del trabajador, especialmente para los profesionales de enfermería quienes son los mas afectados al mantener el contacto directo las 24 horas del día con el paciente y su familia.

A partir de este hecho entidades internacionales como la Organización Mundial de la Salud (OMS), el Consejo Internacional de Enfermería (CIE) y la Organización Internacional del Trabajo (OIT) entre otros, reconocen la importancia que tienen los profesionales de enfermería en el campo de salud, de la misma forma que han identificado las condiciones

\footnotetext{
*Autor de correspondencia: zcogollom@unicartagena.edu.co
} 
en la que estos profesionales laboran, con incremento de la carga laboral, pérdida de seguridad laboral, falta de suministros para la prestación de servicios, bajos salarios, falta de recurso humano de enfermería e incremento de procesos judiciales, lo que conlleva a sobrecarga laboral, cansancio y agotamiento físico-mental (Pico, 2001; Romero et al., 2008; Torres, 2004).

La Enfermería como profesión en Colombia, es facultada a través de la Ley 266 de 1996, para ejercer su práctica dentro de una dinámica interdisciplinaria, multiprofesional y transdisciplinaria. Puede ejercer su práctica en los ámbitos donde la persona vive, trabaja, estudia, se recrea y se desarrolla, y en las instituciones que directa o indirectamente atienden la salud (Congreso de Colombia, 1996). En atención a ello, el campo de actuación del profesional de Enfermería en Colombia es bastante variado, al igual que sus alcances y proyección social, dado por la diversidad de formas en que ésta puede ser ejercida.

La crisis que atraviesa este recurso humano, al igual que los otros profesionales del sector salud, caracterizada por los graves desequilibrios laborales, entornos poco saludables, desinversiones del sector salud, unidas a unas condiciones y políticas de empleo deficientes, están influyendo en la retención de los profesionales, en la productividad, en los resultados de las instituciones de salud, y en último término, en la calidad de los servicios ofrecidos a la población beneficiaria (Consejo Internacional de Enfermería, 2014).

Para el caso particular de Colombia, la Asociación Nacional de Enfermeras (Asociación Nacional de Enfermería de Colombia, 2014), expone que después de la aplicación de la reforma al sistema de salud, la mayoría de profesionales del sector ha visto desmejorado sus condiciones laborales, las formas de contratación y los salarios. Además, se introdujeron nuevas responsabilidades en la gestión de los servicios y de costo, intermediaciones en la contratación laboral e insatisfacción laboral en todo el equipo de salud, de esta manera se merma considerablemente la motivación y la posibilidad de desarrollo de los profesionales. Por otro lado, describen la precarización del trabajo como un retroceso en el desarrollo social, económico y humano, en la medida en que limita toda posibilidad de libertad y dignidad. De igual manera, informan que el profesional de enfermería se enfrenta a formas de contratación que generan pérdida de la relación laboral con las entidades prestadoras de servicios de salud. De este modo, la vinculación a lugares de trabajo se da a través de agentes externos, principalmente las llamadas cooperativas de trabajo asociado. Para Melgarejo \& Romero la promoción de estas modalidades de contratación han llevado a la profesión de enfermería a una pérdida de derechos y beneficios ganados por el reconocimiento al trabajo y al trabajador. Este último se ha convertido en un objeto vulnerable, fácilmente canjeable, por cuanto es muy fácil de prescindir de su labor (Melgarejo y Romero, 2012).

Para Cárdenas la relación inter o multiprofesional que enfrentan las enfermeras es desigual, es decir, el monto económico que se le paga, casi nunca se equipara con los de otros profesionales, lo cual ocasiona que en la sociedad se identifiquen profesionales con más fuerza que otros, asociados a mayor status, prestigio y profesionales débiles con menor prestigio, vinculados a cargos de subordinación (Cárdenas et al., 2009). La reciente profusión de nuevos roles profesionales y los cambios en la práctica de la enfermería están siendo objeto de discusión en el mundo y parece tener impacto en el tipo de cuidados y en el formato de su administración. La práctica de enfermería se está tornando más diversa y las fronteras de la experiencia intra e interprofesional son difusas con relación al significado de su esencia profesional, al objetivo de la práctica, a las expectativas de 
los propios profesionales. Para Mesa el cambio de rol se origina como uno de los efectos negativos de los procesos de modernización, ya que promueven que en las instituciones atribuyan y otorguen funciones y responsabilidades al profesional de enfermería que no hacen parte de su saber-hacer, ni del campo de enfermería; por el contrario, algunas de ellas parecen ser completamente ajenas al objeto disciplinar, es decir, al cuidado de enfermería y de la salud, ello desplaza la relación de cuidado a la persona, que es lo que se pretende establecer con la redefinición del rol (Mesa y Romero, 2010).

Es cierto que los avances científico-técnicos pueden requerir nuevas actitudes y conocimientos por parte del profesional en el desarrollo de su rol, pero estas no se pueden apartar del objeto y los objetivos de la enfermería, porque no se brindaría cuidado a sujetos, sino a objetos. Al respecto, Romero considera que esta situación es una de las tareas urgentes de superar en el ejercicio actual de las enfermeras. La resignificación del acto de cuidado, cuya esencia está en los sujetos, debe ser una estrategia de resistencia ante la adversidad, ya que el nuevo rol aleja al profesional de la relación dialógica con el sujeto de cuidado (Romero, 2007).

Estas condiciones de trabajo guardan estrecha relación con la satisfacción e insatisfacción laboral, por tanto, éste es uno de los factores relacionados con la motivación, indispensable en la calidad del servicio ofertado por la enfermera (Calderon, 1999). El cumplimiento de diferentes funciones aleja a la enfermera cada vez más del cuidado al paciente y conllevan a un deterioro de la identidad profesional; agravado por la falta de agremiación, de solidaridad y de integración del conocimiento ético, político, cultural, científico y legal, para el ejercicio de la profesión (Avendaño y Grau, 1997). El objetivo del presente estudio fue identificar las condiciones de trabajo de los egresados de un programa de Enfermería de una universidad pública la ciudad de Cartagena, Colombia

\section{METODOLOGÍA}

El presente es un estudio descriptivo. La población la constituyeron 110 enfermeras egresadas del Programa de Enfermería, escogidas a través de un muestreo probabilístico aleatorio simple y estratificado por institución.

Los participantes diligenciaron en forma individual y anónima un cuestionario que incluyó las características sociodemográficas, características del cargo desempeñado, relaciones laborales, tipo de contratación y la Escala SL-SPC que es una escala aditiva de medición ordinal de 36 ítems que incluye 7 factores como: los elementos materiales o de infraestructura donde se desenvuelve la labor cotidiana de trabajo, los beneficios laborales, políticas, relaciones sociales, desarrollo profesional, desempeño y relación con la autoridad. Esta escala ha sido utilizada en otros estudios, la validación se llevó a cabo en población latinoamericana, mediante la opinión de seis expertos en la elaboración de escalas con aceptables propiedades psicométricas, valor alfa cronbach (0.70) (Palma, 1999). La aplicación del cuestionario se realizó en las instituciones de salud, durante ese proceso se informó a las participantes sobre los objetivos del estudio, la voluntariedad de la participación y el manejo confidencial de la información.

Una vez diligenciados los datos se incorporaron en una matriz en Microsoft Excel y se procesaron en el programa Statistical Package Socials Sciencies (SPSS) versión 15.0. Se realizo un análisis descriptivo (promedios, distribución de frecuencias, porcentajes y desviación estándar), con Intervalos de Confianza al 95\%. 


\section{RESULTADOS}

Un total de 110 profesionales de enfermería, diligenciaron consistentemente la encuesta. La edad del grupo oscilo entre 23 y 55 años con un promedio de 33.9 años (DS: 8.9). Un cuestionario fue eliminado por mal diligenciamiento de la información, sin que esto restase validez a la muestra.

El 91\%, de los encuestados fueron mujeres, 99,1\% residen en Colombia, el 0,9\% reside en los Estados Unidos y en otros departamento del país, el 48,4\% estaban casados, el $66,6 \%$ son solteras, el 74,6\% no tienen hijos, mientras que el 10,9\% tienen 2 hijos, 95\% estuvieron en el momento afiliados al régimen de salud contributivo.

En cuanto, al tiempo de ejercicio profesional, la población de estudio; el 30\%, tiene de 1 a 6 meses, el 16\% tiene entre 18 y 24 meses, solo un 6\% tiene entre 42 y 48 meses, en cuanto al tiempo de vinculación a la institución, el 50\% dice tener más de 3 años y el otro 50\% menos de un mes (ver Tabla 1):

Tabla 1.

Características sociodemográficas de las enfermeras(os) de Cartagena, Colombia

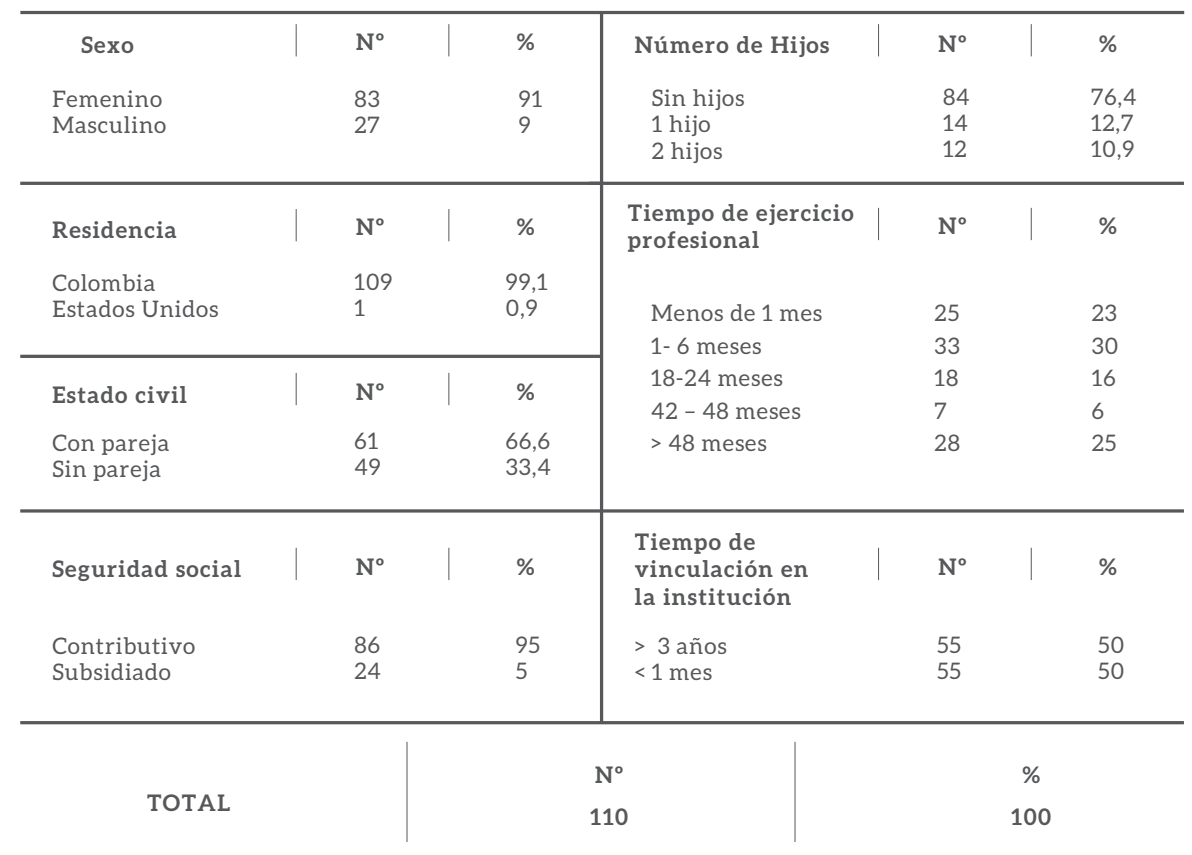

En lo que respecta, a la situación laboral 93\% de los egresados están laborando, de estos el $98 \%$ no realizan otra actividad remunerada y el 94,7\% desea instalar un negocio. Referente al tipo de contratación, el 46\% de las encuestadas tienen contratación a término fijo y $42 \%$ a término indefinido, solo un $7 \%$ laboran por prestación de servicios.

El $86 \%$ de las egresadas se desarrollan actividades propias de la profesión y el 13\% considera que su empleo está relacionado indirectamente con su profesión. En lo que 
respecta al desempeño profesional, el 64\% se desempeña como Enfermeras de servicio, el $4 \%$ como enfermeras rurales e igual porcentaje de docentes y $3 \%$, como auditorias, $55 \%$ reciben salarios mensuales entre $\$ 1^{\prime} 500.000$ y $\$ 2$ '000.000, es decir, de 3 a 4 salarios mínimos mensuales legales vigentes al año 2015, 33\% entre 1'000.000 y menor 1'500.000 y solo un 4\% tiene un sueldo mayor o igual a 3'000.000 y menor a 3'500.000. El 95\% de los egresados considera que el trabajo ha contribuido a su crecimiento personal, el $62 \%$ se encuentra satisfecho con el empleo y el 32\% "Muy satisfecho" (ver Tabla 2):

Tabla 2.

Características laborales de las enfermeras(os) de Cartagena - Colombia.

\begin{tabular}{|c|c|c|c|c|c|}
\hline $\begin{array}{c}\text { Labora } \\
\text { Si } \\
\text { No }\end{array}$ & $\begin{array}{l}\mathrm{N}^{\circ} \\
102 \\
8\end{array}$ & $\begin{array}{l}\% \\
93 \\
7\end{array}$ & $\begin{array}{l}\text { Actividad remunerada } \\
\qquad \begin{array}{c}\mathrm{Si} \\
\text { No }\end{array}\end{array}$ & $\begin{array}{l}\mathbf{N}^{\circ} \\
108 \\
2\end{array}$ & $\begin{array}{l}\% \\
98 \\
2\end{array}$ \\
\hline \multirow{2}{*}{$\begin{array}{l}\text { ¿Desea conseguir un } \\
\text { trabajo o instalar un } \\
\text { negocio? }\end{array}$} & \multirow[t]{2}{*}{$\mathbf{N}^{\circ}$} & \multirow[t]{2}{*}{$\%$} & Tipo de contratación & $\mathbf{N}^{\circ}$ & $\%$ \\
\hline & & & $\begin{array}{l}\text { A termino indefinido } \\
\text { A termino fijo }\end{array}$ & $\begin{array}{l}46 \\
51\end{array}$ & $\begin{array}{l}42 \\
46\end{array}$ \\
\hline \multirow{2}{*}{$\begin{array}{l}\mathrm{Si} \\
\text { No }\end{array}$} & \multirow{2}{*}{$\begin{array}{l}104 \\
6\end{array}$} & \multirow{2}{*}{$\begin{array}{l}94,7 \\
5,3\end{array}$} & $\begin{array}{l}\text { Por prestación } \\
\text { de servicios }\end{array}$ & 8 & 7 \\
\hline & & & Por outsourcing & 6 & 5 \\
\hline Desempeño profesional & $\mathbf{N}^{\circ}$ & $\%$ & Salarios mensuales & $\mathrm{N}^{\circ}$ & $\%$ \\
\hline Enfermeras de servicio & 70 & 64 & 1 у 2 & 36 & 33 \\
\hline Enfermeras rurales & 4 & 4 & 3 у 4 & 61 & 55 \\
\hline Docente & 4 & 4 & Más de 4 & 4 & 4 \\
\hline Auditora & 4 & 4 & $<1$ salario & 9 & 8 \\
\hline Otra & 26 & 24 & & & \\
\hline $\begin{array}{l}\text { Nivel de satisfacción } \\
\text { con su empleo }\end{array}$ & $\mathbf{N}^{\circ}$ & $\%$ & $\begin{array}{l}\text { Utilidad de los } \\
\text { conocimientos }\end{array}$ & $\mathrm{N}^{\circ}$ & $\%$ \\
\hline Satisfecho & 68 & 62 & Muy útil & 91 & 83 \\
\hline Muy satisfecho & 35 & 32 & Útil & 17 & 15 \\
\hline Poco satisfecho & 7 & 6 & Poco útil & 2 & 2 \\
\hline $\begin{array}{l}\text { ¿En su opinión, para } \\
\text { el trabajo que está } \\
\text { desempeñando, } \\
\text { realmente qué nivel } \\
\text { de estudios se requiere? }\end{array}$ & $\mathbf{N}^{\circ}$ & $\%$ & $\begin{array}{l}\text { ¿Volvería a estudiar } \\
\text { en la Universidad } \\
\text { de Cartagena? }\end{array}$ & $\mathbf{N}^{\circ}$ & $\%$ \\
\hline Universitario & 94 & 85 & Si & 101 & 92 \\
\hline Especialización & 16 & 15 & No & 9 & 8 \\
\hline $\begin{array}{l}\text { ¿Cuál sería la principal } \\
\text { razón para querer volver } \\
\text { a este Programa? }\end{array}$ & $\mathbf{N}^{\circ}$ & $\%$ & $\begin{array}{l}\text { ¿En el futuro, le gustaría } \\
\text { cursar otros estudios en } \\
\text { la Universidad de }\end{array}$ & $\mathbf{N}^{\circ}$ & $\%$ \\
\hline $\begin{array}{l}\text { Calidad de la formación } \\
\text { Reconocimiento de la } \\
\text { institución }\end{array}$ & $\begin{array}{l}63 \\
32\end{array}$ & $\begin{array}{l}57 \\
29\end{array}$ & $\begin{array}{l}\text { Cartagena? } \\
\text { Si } \\
\text { No }\end{array}$ & $\begin{array}{l}109 \\
1\end{array}$ & $\begin{array}{l}99 \\
1\end{array}$ \\
\hline Calidad de los profesores & 9 & 8 & & & \\
\hline $\begin{array}{l}\text { Posibilidad de encontrar } \\
\text { empleo rápidamente }\end{array}$ & 7 & 6 & & & \\
\hline $\begin{array}{l}\text { ¿Principalmente, qué } \\
\text { otros estudios le gustaría } \\
\text { cursar en la Universidad } \\
\text { de Cartagena? }\end{array}$ & $\mathrm{N}^{\circ}$ & $\%$ & $\begin{array}{l}\text { ¿Recomendaría a un } \\
\text { bachiller seleccionar el } \\
\text { programa de Enfermería } \\
\text { de la Universidad de }\end{array}$ & $\mathbf{N}^{\circ}$ & $\%$ \\
\hline $\begin{array}{l}\text { Diplomados } \\
\text { Universitarios } \\
\text { Especialización } \\
\text { Maestría } \\
\text { Ninguno }\end{array}$ & $\begin{array}{l}4 \\
7 \\
86 \\
14 \\
4\end{array}$ & $\begin{array}{l}4 \\
6 \\
77 \\
13 \\
4\end{array}$ & $\begin{array}{l}\text { Cartagena? } \\
\mathrm{Si}\end{array}$ & 110 & 100 \\
\hline TOTAL & & & $\begin{array}{l}N^{\circ} \\
110\end{array}$ & & \\
\hline
\end{tabular}


En lo concerniente, a la utilidad de los conocimientos, habilidades y destrezas aprendidas en su carrera tanto para el desempeño laboral como en otros aspectos de su vida, el $83 \%$ las considera "Muy útil" y solo un $2 \%$ poco útil, el $85 \%$ considera que para desempeñarse solo se requiere el nivel Universitario, mientras que el 15\% cree importante hacer una especialización.

En lo que tiene que ver con la identidad profesional, el 92\% considero que volvería a estudiar Enfermería en la Universidad de Cartagena y la principal razón para volver a este programa es por la Calidad de la formación en un 57\%, el 77\% le gustaría en el futuro hacer una especialización, y el 13\% maestría y el 100\% le recomendaría a un bachiller estudiar esta carrera.

\section{DISCUSIÓN}

Los resultados del presente estudio arrojaron, que la mayoría de los participantes 91\% son mujeres. Estos resultados son similares a los encontrados por Castillo et al. (2014), en el cual se estudiaron 156 profesionales de enfermería, donde el 94,2\% (147) fueron mujeres. Asimismo, señalan que las mujeres constituyen la mayor fuerza laboral en Enfermería, dado que tradicionalmente en la sociedad, el cuidar es identificado como una actividad inherente a la mujer.

En este estudio el $46 \%$ de las enfermeras se encuentran vinculadas por contratos a término fijo y el $42 \%$ a término indefinido. En estudios previos, la vinculación laboral de los trabajadores estudiados, el tipo de contratación más común es a término fijo (70.5\%) (Castillo et al. 2014). En este mismo sentido, la Asociación Nacional de Enfermeras de Colombia (ANEC), según datos del mes de octubre de 2014 de la Unidad de Registro Profesional, informó que el 57\% de las enfermeras tienen vinculación flexible (Carvallo, 2014).

Referente al salario, el $45 \%$ reciben salarios mensuales entre $\$ 1^{\prime} 500.000$ y $\$ 22^{\prime} 000.000$, es decir, de 3 a 4 salarios mínimos mensuales legales vigentes al año 2016, el 33\%, un porcentaje alto entre 1'000.000 y menor 1'500.000 y solo un 4\% tiene un sueldo mayor o igual a 3'000.000 y menor a 3'500.000, porcentaje superior al reportado por ANEC en el 2014 donde el $47 \%$ devenga entre $\$ 500.000$ y $\$ 1.500 .000$ (Carvallo, 2014). En un estudio similar Cogollo et al. (2010) informó un rango salarial entre $\$ 1$ '384.500 y $\$ 1$ '846.000 lo que significa, que 6 años después, el profesional continua recibiendo el mismo valor como pago por sus servicios.

Al respecto vale la pena destacar, según la Encuesta Calidad de Vida (DANE, 2014), en el año 2017, para una familia de ingresos promedio, el valor de la canasta básica familiar tenía un precio mensual de 1.300 .000 pesos; en contraste, el SML estaba en 737.717 pesos, de este modo, se requieren 2 salarios mínimos legales -SML-para adquirir parte de la canasta básica familiar. Esto quiere decir, que el salario que hoy reciben las enfermeras solo alcanza a cubrir la canasta familiar, lo que conlleva a que las necesidades de formación y cualificación no se puedan satisfacer, con imposibilidad de mantenerse a la altura de las exigencias, como tampoco representa un ingreso profesional, muchas veces especializado, con experiencia y experticia, agravada esta situación por el tipo de vinculación terciarizada (DANE, 2014) y que repercute en la falta de motivación por parte de los profesionales de enfermería para continuar con la formación. Al respecto, el 
85\% de los profesionales de enfermería de este estudio piensa que para desempeñarse solo se requiere el nivel Universitario y que las habilidades y destrezas aprendidas en su carrera son útiles para su desempeño laboral.

En la medida que los sueldos no son justos, ni acordes con las expectativas, las exigencias del puesto de trabajo, el nivel de habilidades individuales, ni con los niveles de sueldo de los otros profesionales del mismo entorno laboral, es posible que haya insatisfacción y desmotivación (20). Al respecto el CIE (Consejo Internacional de Enfermeras) señala que el acceso equitativo a los necesarios servicios de salud de buena calidad no puede conseguirse sin un número adecuado de enfermeras preparadas idóneamente (Consejo Internacional de Enfermería, 2014).

Otra variable que se relaciona con la erogación del salario es el número de personas a cargo, en este estudio el 66,6\% de las enfermeras son solteras, el 74,6 \% no tienen hijos, mientras que el 10,9\% tienen 2 hijos, lo que reduce las posibilidades de satisfacción de necesidades básicas para el grupo familiar y genera frustración en el trabajador por las escasas posibilidades para cumplir con exigencias familiares. En este sentido, la literatura especializada plantea, que en términos generales, existe una relación positiva entre la satisfacción de los empleados y el salario recibido, así mismo, la satisfacción de los profesionales de la salud en el trabajo es uno de los indicadores que condicionan la calidad asistencial (Perezagua et al., 2005). Para Robbins la satisfacción en el trabajo está determinada por el atractivo de la tarea, las recompensas asociadas, las relaciones con los compañeros de trabajo y las condiciones laborales a las que los empleados están expuestos (Robbins, 1987). Gargallo Castel (2008). En un estudio sobre la satisfacción laboral y sus determinantes en las cooperativas, informó que el 60\% de los socios y casi un 55\% de los no socios consideraron que es la retribución, el principal factor motivador de la satisfacción laboral.

Los trabajadores insatisfechos faltan al trabajo con más frecuencia, incurren en retrasos, suelen abandonar la empresa más a menudo para la que trabajan, además de inducir otros comportamientos organizativos inadecuados y el incremento del índice de accidentes de trabajo.

Enfermería tiene una relevancia en campo de la salud en el proceso de cuidado de la salud, sin embargo, las condiciones laborales, salariales y el reconocimiento social, no son congruentes con la dedicación y el profesionalismo que implica el acto de cuidar. Vale la pena destacar y comprender la importancia del entorno laboral en momentos de cambio significativo cuando los servicios se deben rediseñar en armonía con el nuevo modelo de salud MIAS. Muchos profesionales de enfermería requieren de más formación y preparación para enfrentar nuevas funciones con el fin de responder de manera eficaz. Cada vez más, las enfermeras requieren desempeñar nuevos roles; de dirección, de política y retomar el cuidado directo en las instituciones de salud, todos los cuales crean entorno laboral diferente y una diferente cultura asociada con ellos (Consejo Internacional de Enfermería, 2014).

Es lamentable ignorar la función vital que las enfermeras han de desempeñar, las enfermeras necesitan ser reivindicadas por el ministerio de salud y el gobierno en general como un colectivo mayoritariamente femenino y que solo por esto, tiene la desventaja de menores salarios frente a otros profesionales de la salud que son mayoritariamente masculinos, se requiere de manera urgente reducir las desigualdades entre mujeres y varones. Así mismo, las enfermeras requieren empoderarse de sus agremiaciones y trabajar de manera conjunta y activa para defender los derechos laborales, tener puestos 
de trabajos dignos, mejorar la formación, cambiar la imagen profesional y participar en todos los niveles de política y en los espacios de toma de decisiones (Consejo Internacional de Enfermería, 2014).

\section{CONCLUSIONES}

Se concluye que en general, las enfermeras presentan condiciones de trabajo deficientes no solo en materia de retribuciones, carga laboral y personal de apoyo, sino que además, las condiciones de infraestructura física de los puestos de trabajo, así como de materiales y equipos en el lugar de trabajo son percibidos como deficientes.

\section{REFERENCIAS BIBLIOGRÁFICAS}

Acevedo G, Farias A, Sánchez J, Astegiano C, Fernández A. (2012). Condiciones de trabajo del equipo de salud en centros de atención primaria desde la perspectiva de trabajo decente. Revista Argentina de Salud Pública, 3(12):15-22.

Asociación Nacional de Enfermería de Colombia (2014). Enfermería, una fuerza para el cambio. Recuperado de: http://www.anec.org.co/revista77/revista\%2077.pdf

Avendaño C., Grau P. (1997). Riesgos para la salud de las enfermeras del sector público de Chile. Rev. Enf., 102: 15-26.

Calderón A. (1999). Estudio de las posibles relaciones entre el absentismo laboral y el grado de insatisfacción profesional en la administración pública. Rev. Mapfre Medicina, 10 (1): 23-27

Cárdenas, L; Arana, B; Monroy, A; García, M. (2009). La autonomía profesional en Enfermería. En: Cuidado profesional de Enfermería. México: Federación Mexicana de Asociaciones de Facultades y Escuelas de Enfermería (FEMAFEE).

Carvallo B. (2014). Principales indicadores socio laborales de la Profesión de Enfermería en Colombia. Publicación oficial de la Asociación Nacional de Enfermeras de Colombia, ANEC.

Castillo Ávila I., Torres Llanos N., Ahumada Gómez A., Cárdenas Tapias K., Licona Castro S. (2014). Estrés laboral en enfermería factores asociados, Cartagena (Colombia). Salud Uninorte. 30(1): $34-43$

Cogollo Z., Gómez E. (2010). Condiciones laborales en enfermeras de Cartagena, Colombia. Avances en Enfermería, 23 (1).

Congreso de Colombia (1996). Ley 266 de enero 25 de 1996, Diario Oficial No. 42710 de 1996.

Consejo Internacional de Enfermería (2014). Las Enfermeras una fuerza para el cambio. Consultado el 15 de julio de 2015. Recuperado de: http://www.consejogeneralenfermeria.org/docs_ revista/DIE2014.pdf

DANE (2014). Encuesta Calidad de Vida. Recuperado de: https://www.desdeabajo.info/.../27125encuesta-calidad-de-vida-dane-colombia-2014

Fernández B, Paravic T (2003). Nivel de Satisfacción Laboral en Enfermeras de Hospitales Públicos Y Privados de la Provincia de Concepción, Chile. Cienc. Enferm., 9 (2): 57-66.

Gargallo Castel, A. F. (2008). La satisfacción laboral y sus determinantes en las cooperativas. Management, 563-575 
Melgarejo L., Romero M. (2012). Profesionales de enfermería y cuidado en las condiciones laborales actuales. Investigación en Enfermería: Imagen y Desarrollo, 2(2):55-92.

Mesa l., Romero M. (2010). Profesionales de enfermería y cuidado en las condiciones laborales actuales. Investigación en Enfermería: Imagen y Desarrollo, 12(2). Recuperado de: www. javeriana.edu.co/Facultades/Enfermeria/revista1/files/132.pdf

Palma S. (1999). Elaboración y Validación de una Escala de Satisfacción Laboral SL-SPC para trabajadores de Lima Metropolitana. Revista Teoría e Investigación en Psicología, 9(1):27-34.

Perezagua, M., Moro, O., Vidal, M., Villal, M. (2005). Satisfacción personal de enfermería. ¿Se cumplen nuestras expectativas?. Sociedad Española de Enfermería de Urgencias y Emergencias, 35

Pico Merchán M. (2001). Metodología de los panoramas de los factores de riesgo ocupacional: estrategia educativa en salud ocupacional. Hacia la promoción de la salud., 6:17-24

Robbins S. (1987). Comportamiento organizacional. Conceptos, controversias. Prentice-Hall Hispanoamericana S.A. México.

Romero M. (2007). El cuidado de enfermería en la adversidad: compromisos de resistencia desde la docencia, Av Enferm.,15(2):112-23.

Romero M., Mesa L., Galindo S. (2008). Calidad de vida de las enfermeras y sus consecuencias para el cuidado. Avances en enfermería, 26 (2): 59-70

Torres J. (2004). Reflexiones sobre funciones del personal de enfermería. Rev. Cubana Salud Pública, 30(4). Recuperado de: http://scielo.sld.cu/scielo=S0864-3466=2004000400009\&lng $=e s \& n r m=i s o$ 